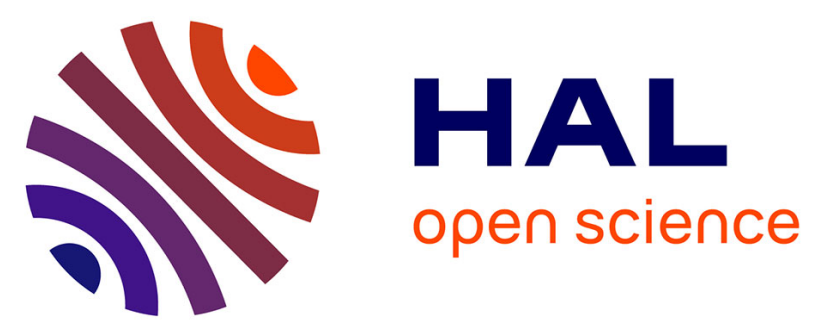

\title{
Development and validation of an OFDM/DVB-T sensor for positioning
}

Damien Serant, Olivier Julien, Christophe Macabiau, Paul Thevenon, Stéphane Corazza, Mathieu Dervin, Marie-Laure Boucheret, Lionel Ries

\section{- To cite this version:}

Damien Serant, Olivier Julien, Christophe Macabiau, Paul Thevenon, Stéphane Corazza, et al.. Development and validation of an OFDM/DVB-T sensor for positioning. IEEE/ION PLANS 2010, Position Location and Navigation Symposium, May 2010, Indian Wells, United States. pp 988-1001, 10.1109/PLANS.2010.5507273 . hal-01022200

\section{HAL Id: hal-01022200 \\ https://hal-enac.archives-ouvertes.fr/hal-01022200}

Submitted on 30 Sep 2014

HAL is a multi-disciplinary open access archive for the deposit and dissemination of scientific research documents, whether they are published or not. The documents may come from teaching and research institutions in France or abroad, or from public or private research centers.
L'archive ouverte pluridisciplinaire HAL, est destinée au dépôt et à la diffusion de documents scientifiques de niveau recherche, publiés ou non, émanant des établissements d'enseignement et de recherche français ou étrangers, des laboratoires publics ou privés. 


\section{Development and validation of an OFDM/DVB-T sensor for positioning}

\author{
Damien Serant \\ ENAC/TéSA \\ Toulouse, France \\ serant@recherche.enac.fr \\ Paul Thevenon \\ ISAE/TéSA \\ Toulouse, France
}

Marie-Laure Boucheret

University of Toulouse, IRIT/ENSEEIHT/TéSA

Toulouse, France

\author{
Olivier Julien, Christophe Macabiau \\ ENAC \\ Toulouse, France
}

\author{
Stéphane Corazza, Mathieu Dervin \\ Thales Alenia Space \\ Toulouse, France
}

\author{
Lionel Ries \\ CNES \\ Toulouse, France
}

\begin{abstract}
The use of Global Navigation Satellite System (GNSS) for positioning has grown significantly in recent years thanks in particular to the development of several mass-market applications, such as car navigation or mobile positioning. Unfortunately, in difficult environments such as dense urban or indoor areas, GNSS exhibits degraded performances in terms of precision and availability. The use of signals of opportunity is one of the solutions to replace or assist GNSS in those environments. These signals are communication signals that are usually designed to provide a service in dense environment and can thus be used in location where GNSS is unavailable. Several commercial positioning services based on signals of opportunity already exist such as ROSUM with ATSC digital TV signals, or Skyhook with Wi-Fi signals
\end{abstract}

This paper investigates the potential of the digital TV European (but used worldwide) standard DVB-T, based on an OFDM air interface, to supply a navigation service. To support a previously proposed DVB-T receiver architecture, a DVB-T receiver simulator is presented that includes the principal blocks required for communication with enhanced navigation-oriented functionalities. In particular, this simulator includes a Delay Lock Loop (DLL) for accurate synchronization purpose.

The theoretical expressions of pseudo-range sigma error and tracking threshold in case of Line-Of-Sight (LOS) signal are proposed and compared to simulation results, in order to validate the DLL module of the simulator. Then, performances in a more realistic Rayleigh multipath channel are presented. This paper also proposes description and explanations of impairments due to absence of LOS signal in urban environment and points out the limits of existing urban channel propagation models for a positioning solution. $D V B-T$

Keywords - signal-of-opportunity, TV-positionning, OFDM,

I would like to thank CNES and Thales Alenia Space France for funding this work

\section{INTRODUCTION}

Global Navigation Satellite System (GNSS) has grown significantly in recent years thanks to the development of many mass-market applications, such as car navigation, asset management or mobile positioning. However, in difficult environments such as dense urban or indoor areas, GNSS shows only limited performances in terms of accuracy and availability due to masking and/or reflections and/or diffraction of the direct signal. This compromises the use of GNSS as a standalone mean for robust positioning in challenging environments. Fortunately, urban areas have the advantage to have dense telecommunication networks to provide mobile phone, $\mathrm{TV}$, and all sorts of services to the user. The use of these signals is now strongly considered as a way to provide a rough estimation of the user position when GNSS is not available, or to assist GNSS in those environments. This is why these signals are often referred to as signals of opportunity. They include Wi-Fi, WiMAX, TV signals, mobile phone signals, etc... Several commercial navigation services based on signal of opportunity already exist : Rosum uses Time Difference of Arrival (TDOA) with ATSC digital TV signals [1], Skyhook uses fingerprinting with Wi-Fi signals [2], etc...

The study presented in the paper targets the use of the European Digital Video Broadcast - Terrestrial (DVB-T) signals for positioning. This digital TV standard, although developed in Europe, has been widely accepted worldwide [3] and is the basis for other standards targeting handheld receivers, DVB-H (Handheld) and DVB-SH (Satelitte-toHandheld). The DVB-T signal uses an Orthogonal Frequency Division Multiplexing (OFDM) air interface. The advantages of TV signals for our purpose, especially when designed for handheld devices, are: 
- The transmitter density is high and any receiver within TV coverage should be able to process at least one signal from one transmitter.

- the received signal power level is high enough to authorize an indoor or dense urban service area reception (which is often impossible with GNSS signals). Moreover, it is easy to understand that the typical received signal to noise ratio threshold for a communication service will likely be significantly higher than that required for ranging due to necessity of demodulating the communication message with a low Bit Error Rate (BER).. Therefore, as the broadcasting network has been designed to provide at least one emitter with power above the demodulation threshold, it will be likely that several emitters of this same network will be "available" for ranging.

- $\quad$ the wide bandwidth of TV signals (between 6 and 8 $\mathrm{MHz}$ ), larger than current mass-market GNSS signals, can potentially offer a better synchronization capability than the current GPS C/A.

- $\quad$ DVB-T signals (and all OFDM signals) contain pilot symbols that are used for equalization and can be used for synchronization. Those pilots can be used to construct a Delay Lock Loop (DLL).

An example of DVB-based ranging architecture was presented in earlier work [4]. This article presented the DLL based on DVB-T pilots. To complement this, the present paper introduces a DVB-T software receiver including, in a same architecture, the classical signal processing functions necessary to provide a communication service and the navigation signal processing functions for ranging, mostly based on the above-mentioned DLL structure to provide a navigation service.

To prove the suitability of the tracking structure, and to participate to the modeling of the pseudo-distance measurements coming from the DVB-T sensor, this paper also investigates theoretically the impact of thermal noise on the tracking algorithm. This represents the ideal case of the reception of the direct signal alone. However, the DVB-T using a terrestrial network of transmitter, the propagation channel is almost always strongly affected by reflections/diffractions/masking. Consequently, the effect of multipath is also analyzed to quantify its impact on pseudorange error.

This paper is organized as follow:

- The first section describes the principles of OFDM modulation and its related processing. This section also presents the DVB-T standard, including the signal parameters and the pilot structures.

- The second section deals with the classical DVB-T receiver architecture. It describes the structure of the communication part of the developed DVB-T software receiver, regrouping the classical synchronization functions of an OFDM receiver.

- The third section presents the navigation module of the developed DVB-T receiver. In a first part the structure of the navigation part of the software receiver is presented, with in particular the pseudorange computation principle. The second part deals with the performance of the delay tracking by proposing theoretical expressions of the pseudorange error sigma and of the tracking threshold validated by simulation. The impact of multipath on the pseudorange estimation is also considered in the last part.

- Finally the last section presents the performance of the proposed ranging method in a more realistic multipath channel.

\section{ORTHOGONAL FREQUENCY DIVISION MULTIPLEXING} AND DIGITAL VIDEO BROADCASTING

\section{A. Orthogonal Frequency Division Multiplexing}

The concept of OFDM [5] consists in transmitting in parallel $N$ complex data symbols over $N$ orthogonal narrowband subcarriers (1 subcarrier carries 1 symbol). The width of these subcarrier is chosen narrow enough so that the channel frequency response can be considered as flat over the subcarrier bandwidth. The consequence is that channel equalization becomes very simple even in case of dense multipath environment. Thanks to the orthogonality of the subcarriers, their spectra can overlap without interfering with each other, allowing an excellent spectral efficiency and no Inter-Carrier Interference (ICI) when the receiver is synchronized.

An OFDM useful symbol is obtained by passing the $\mathrm{N}$ complex data symbols through an inverse-Fast Fourier Transform (iFFT) operator. The useful part of the OFDM symbol is thus composed of $\mathrm{N}$ samples. To take advantage of the FFT algorithm, $\mathrm{N}$ is generally chosen to be a power of two. Conversely, the demodulation of an OFDM symbols is perform by a direct FFT.

Additionally, a guard interval of $N_{C P}$ samples is inserted before the useful OFDM symbols in order to avoid InterSymbol Interference (ISI). In the case of DVB, this guard interval, referred to as Cyclic Prefix (CP) is a replica of the last $N_{C P}$ samples of the OFDM symbol. By doing so, any demodulation of the OFDM symbol that is done with an Nsample FFT starting in the CP will only result in a phase rotation of each subcarrier proportional to its frequency offset, thus easily equalized. An OFDM symbol is thus composed of $N+N_{C P}$ samples.

To illustrate the OFDM modulation principle, Fig. 1 shows an OFDM transmission block diagram. 


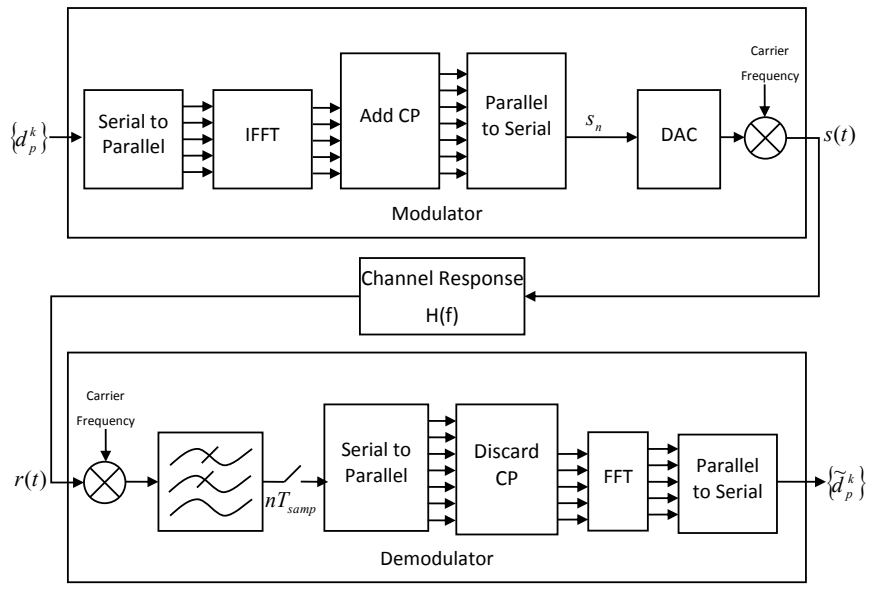

Figure 1. OFDM transmission block diagram

According to this diagram, the expression of the n-th sample of the k-th emitted OFDM symbol is:

$$
\begin{gathered}
s_{n}^{k}=\frac{1}{N} \sum_{p=0}^{N-1} d_{p}^{k} \exp \left(j 2 \pi \frac{p n}{N}\right)=i F F T\left(d_{p}^{k}\right)[n] \\
\text { with }-N_{C P} \leq n \leq N-1
\end{gathered}
$$

where:

- $n$ is the sample time index,

- $\quad p$ is the subcarrier index,

- $\quad k$ is the OFDM symbol number,

- $d_{p}^{k}$ is the $\mathrm{p}$-th complex data symbol of the k-th OFDM symbol and

- $\quad s_{n}^{k}$ is the n-th sample of the k-th OFDM symbol.

The sampled received signal is affected by several impairments:

- $\quad$ Carrier Frequency Offset (CFO), noted $\Delta f$ that occurs when the receiver local oscillator (LO) used for down conversion does not match with the carrier of the received signal. It results in two factors: frequency mismatch between emitter and receiver LO, and the resulting Doppler effect of due to the movement of the receiver and/or the emitter. It is usually normalized by the frequency distance between 2 subcarriers $\left(1 / T_{U}\right)$. By construction $T_{U}$ is also the duration of the useful part of an OFDM symbol (the $N$ samples). This CFO can be decomposed into an integer and a fractional part so that $\Delta f=\delta f+p_{f}$, with $\delta f \in[-0.5,0.5[$ and $p_{f} \in \mathbb{N}$.

- $\quad$ Sampling Clock Offset (SCO), noted $\delta_{S C O}$, is, due to sampling clock frequency mismatch between emitter and receiver and Doppler effect. Its value is expressed in part per million (ppm), which corresponds to a clock drift of $1 \mu$ s per second.

- Timing Offset, noted $\Delta \tau$, corresponding to the time difference between the supposed and the real beginning of one OFDM symbol normalized by the sampling period. This means that the FFT made by the receiver is not perfectly synchronized with the OFDM useful symbol.

- Distortion of the channel with a frequency response $H(f)$ and impulse response $h(t)$

- $\quad$ Additive White Gaussian Noise (AWGN) $n(t)$

Thus the received signal is:

$$
r(t)=(s(t) * h(t))\left(t-\Delta \tau \cdot T_{\text {samp }}\right) \mathrm{e}^{j 2 \pi \frac{\Delta f}{\mathrm{~T}_{U}} t}+n(t)
$$

This received signal is sampled at the sampling period $\left(1+\delta_{S C O}\right) . T_{\text {samp }}$, where $T_{\text {samp }}$ denotes the required sampling period, and grouped by $N+N_{C P}$ samples to form the vectors $\left\{r_{p}^{k}\right\}_{p \in\left[-N_{C P} N-1\right], k \in \mathbb{N}}$.

Taking into account all these impairments and supposing that the FFT windows taken by the receiver begin in the CP and after the end of the last replica of the previous OFDM symbol (i.e. no Inter-Symbol-Interference), the p-th demodulated subcarrier of the $\mathrm{k}$-th received OFDM symbol is [6]:

$$
\begin{aligned}
\widetilde{d_{p}^{k}}= & F F T\left(\left(r_{n}^{k}\right)_{n=0}^{N-1}\right)[p] \\
= & d_{\tilde{p}}^{k} \cdot \alpha\left(\delta f+\delta_{S C O} \tilde{p}\right) \cdot H_{\tilde{p}}^{k} \cdot \exp \left(j 2 \pi \frac{\Delta \tau \tilde{p}}{N}\right) \times \\
& \exp \left(j \phi_{k}\left(\Delta f+\delta_{S C O} \tilde{p}\right)\right)+I C I+n_{p}^{k}
\end{aligned}
$$

where:

- $\tilde{p}=\left(p+p_{f}\right) \bmod N$ the subcarrier index circularly rotated as a result of the integer CFO.

- $\widetilde{d_{p}^{k}}$ is the demodulated symbol over the $\mathrm{p}$-th subcarrier of the k-th OFDM symbol,

- $n_{p}^{k}$ is the noise on the $\mathrm{p}$-th subcarrier of the $\mathrm{k}$-th OFDM symbol,

- $H_{p}^{k}$ is the channel frequency response over the $\mathrm{p}$-th subcarrier of the k-th OFDM symbol,

- $\alpha(x)=\frac{\sin c(\pi x)}{\sin c\left(\frac{\pi x}{N}\right)} \exp \left(\pi x \frac{N-1}{N}\right)$ is the complex attenuation due to $\mathrm{SCO}$ and $\mathrm{CFO}$,

- $\phi_{k}(x)=2 \pi \frac{\left(k\left(N+N_{C P}\right)+N_{C P}\right)}{N} x$ is the phase rotation due to the SCO and CFO and depending on the OFDM symbol number $k$, and

- $\quad$ ICI the inter-carrier interference

According to (3) a demodulated symbol is equal to the transmitted one if the integer part of the CFO is equal to zero (if not, a different transmitted symbol is demodulated instead), and if it is correctly phase-compensated by some complex factors dependent on the different aforementioned impairments. The integer $\mathrm{CFO}$ needs to be estimated and corrected in order to recover the transmitted symbols in the right order. The complex factor can be easily corrected by the channel equalization module. However fractional CFO and SCO induce also ICI which can be considered as noise. 
Consequently those errors need to be corrected before demodulation in order to limit the noise on the demodulated symbols. In AWGN channel and for a high SNR, the degradation of SNR due to ICI (rising from the uncompensated $\mathrm{CFO}$ and $\mathrm{SCO}$ ) is [7],[8]:

$$
D_{I C I}^{d B}(p) \approx 10 \cdot \log _{10}\left(1+\frac{\pi^{2}}{3} S N R \cdot\left(\delta f+\delta_{S C O} p\right)^{2}\right)
$$

The fractional CFO may often reach its maximum value of 0.5 and then the SNR degradation can be several tens of $\mathrm{dB}$. Thus the estimation and the correction of the fractional CFO need to be done before the demodulation in order to limit the induced ICI. The classical values of the SCO (about $10 \mathrm{ppm}$ ) induces a limited SNR degradation (some decibels). Thus, the estimation of the SCO can be done after demodulation and for small values of SCO (less than about $5 \mathrm{ppm}$ ) the correction is not necessary since the SNR degradation is negligible.

\section{B. Digital Video Broadcasting - Terrestrial}

The DVB-T [9] is a European standard for digital TV broadcasting to fixed receivers in the VHF and UHF bands. DVB-H (Handled) and DVB-SH (Satellite-to-Handled), both based on DVB-T, target mobile TV receivers and other potential frequency bands.

These standards define several modes which depend on three parameters: the FFT size $(N)$, the ratio between the Cyclic Prefix length and the useful OFDM symbol length $\left(C P=N_{C P} / N\right)$ and the sampling period $\left(T_{\text {samp }}\right)$. Table I shows different possible values defined in the DVB-T standard for each parameters. The $N$ subcarriers of the useful OFDM symbol have different natures:

- Null subcarriers which are situated on the edges of the signal spectrum and have a zero value. They serve as guard bands to avoid out-of-band emissions of the OFDM signal.

- Data subcarriers which are QAM-modulated and have their amplitude normalized in such a way that their variance is unitary.

- Transmission Parameter Signaling (TPS) subcarriers which are BPSK-modulated and carry information about the actual transmission parameters.

- Pilot subcarriers which are BPSK-modulated. Their value is given by a known Pseudo-Random Binary Sequence (PRBS). Their amplitude is boosted by a factor of $4 / 3$ compared to Data and TPS subcarriers. Pilot subcarriers are used for several synchronization functions and for channel estimation.

The pilot subcarriers can be of two types: continuous pilots

TABLE II. DVB-T PARAMETERS

\begin{tabular}{|l|l|}
\hline Parameters & Possible values \\
\hline $\boldsymbol{N}$ & 2048 (Mode 2K), 4096 (Mode 4K) and 8192 (Mode 8K) \\
\hline $\boldsymbol{C P}$ & $1 / 32,1 / 16,1 / 8,1 / 4$ \\
\hline $\boldsymbol{T}_{\text {samp }}$ & $\begin{array}{l}7 / 64 \mu \mathrm{s}(8 \mathrm{MHz} \text { channels) } 1 / 8 \mu \mathrm{s}(7 \mathrm{MHz}), 7 / 48 \mu \mathrm{s}(6 \\
\mathrm{MHz}) \text { and } 7 / 40 \mu \mathrm{s}(5 \mathrm{MHz})\end{array}$ \\
\hline
\end{tabular}

and scattered pilots. Continuous pilots are always located on the same subcarriers whatever the OFDM symbol. On the contrary scattered pilots are inserted every 12 subcarriers and the first pilot subcarrier index takes four different values $(3,6,9$ or 12) depending on the OFDM symbols number. Therefore the scattered pilot pattern repeats every 4 OFDM symbols. This organization is illustrated in Fig. 2.

The developed DVB-T software receiver can be divided in two part:

- the "communication part" which includes classical functions of an OFDM receiver, and is presented in the next section, and

- the "navigation part" which contains functions dedicated to navigation that are not required for communication application which will be introduced in the fourth section.

\section{STRUCTURE OF THE COMMUNICATION PART OF THE DEVELOPPED DVB-T SOFTWARE RECEIVER}

The "communication part" of the software receiver works in two modes: (1) an initialization mode, which has to be done before the demodulation of data symbols, and (2) an operating mode where data symbols are demodulated while keeping a correct synchronization.

\section{A. Functions in intialization mode}

The initialization mode aims at (1) finding the beginning of the useful OFDM symbol to correctly place the FFT window, (2) finding the fractional CFO, (3) detecting which pattern of the scattered pilot sequence is used in the current symbols and (4) detecting the integer CFO. For these purposes, the following algorithms are used in the developed software receiver:

Cyclic Prefix synchronization, based on the Van de Beek algorithm [10]: use the redundancy of the CP by correlating two groups of $N_{C P}$ samples spaced by $\mathrm{N}$ samples. The expression of the correlation function is given in (5).

$$
\gamma(m)=\sum_{n=0}^{N_{C P}-1} r_{n+m}^{k} \cdot\left(r_{n+\mathrm{m}+N}^{k}\right)^{*}
$$

The absolute maximum of the correlation provides the location of the beginning of the OFDM symbols and the argument of $\gamma$ at this maximum gives an estimation of the fractional part of the CFO. This is described in the following equations.

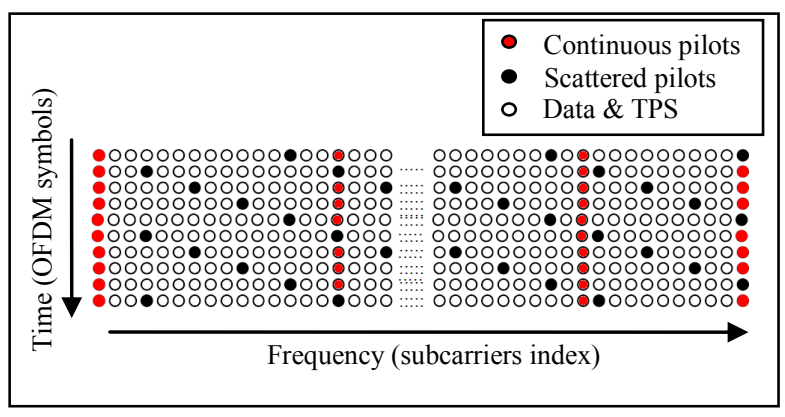

Figure 2. DVB-T pilot organization 


$$
\hat{\tau}=\arg \max _{\mathrm{m}}|\gamma(\mathrm{m})|^{2} \text { and } \widehat{\delta f}=-\frac{1}{2 \pi} \operatorname{angle}(\gamma(\hat{\tau}))
$$

Fig. 3 and Fig. 4 show an example of the functions $|\gamma(\mathrm{m})|^{2}$ and $-\frac{1}{2 \pi} \operatorname{angle}(\gamma(\mathrm{m}))$ for $N=2048, N_{C P}=256$, $\Delta \tau=1000, \delta f=0.25$, and $S N R=15 \mathrm{~dB}$.

In real environment, the estimated peak of $|\gamma(\mathrm{m})|^{2}$ may be strongly affected by multipath. It is then recommended to assign the beginning of the FFT window a few tens of samples before the peak so as to be sure to be in the CP.

After this stage, the FFT demodulation will provide only limited ISI or ICI.

Scattered pilot sequence detection algorithm [6]: after FFT, make the correlation between the scattered pilot subcarriers of the useful OFDM symbol and the scattered pilot subcarriers of the useful OFDM symbol located 4 symbols latter (they should have the same scattered pilot organization as explained in section II.B) assuming the four possible sets of scattered pilot subcarriers. The computed function is:

$$
S(m)=\sum_{p \in \mathcal{P}_{S}(m)} \widetilde{d_{p}^{k}}\left(\widetilde{d_{p}^{k+4}}\right)^{*}, m \in\{0,1,2,3\}
$$

Where $\mathcal{P}_{S}(m)$ is the set of indexes of the m-th pattern of scattered pilot subcarriers.

The maximum value between the four correlations gives the scattered sequence number of the current OFDM symbol:

$$
\widehat{m}=\arg \max _{s}|S(m)|
$$

$\widehat{m} \in\{0,1,2,3\}$ denotes the estimated scattered sequence number.

\section{Estimation of the integer part of CFO [6]}

Integer CFO causes a circular shift of subcarrier with a amount corresponding to the value of the integer CFO. The correlation function of (9), between a set of subcarriers of two consecutive OFDM symbols can permit to estimate the integer CFO.

$$
F(g)=\sum_{p \in \mathcal{P}_{C}} \tilde{d}_{p+g}^{k}\left(\tilde{d}_{p+g}^{k+1}\right)^{*}
$$

where $\mathcal{P}_{C}$ denotes the set of continuous pilot indexes. The integer $\mathrm{CFO}$ is found by looking for the maximum of this correlation function:

$$
\widehat{p_{f}}=\arg \max _{g}|F(g)|
$$

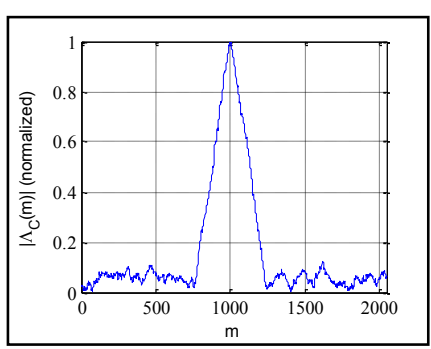

Figure 3. $|\gamma(\mathrm{m})|^{2}$

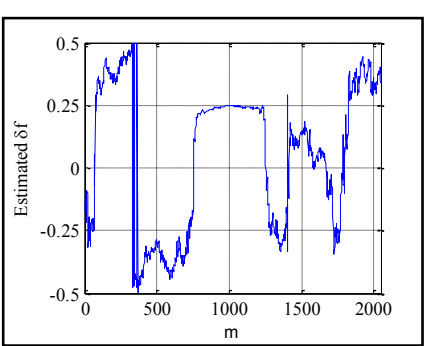

Figure 4. $-\frac{1}{2 \pi} \operatorname{angle}(\gamma(\mathrm{m}))$
This estimator exposes good performance if the channel can be considered constant over two consecutives symbols. In fast varying channels, performances are degraded. The integer CFO can be corrected indifferently before FFT by complex multiplication or after FFT by circularly shifting the subcarrier by $-\widehat{p_{f}}$. mode.

Fig. 5 illustrates the different functions of the initialization

\section{B. Functions in operating mode}

Once the synchronization has been initialized, data demodulation can be started. However, the value of the CFO can change compared to the value found in the initialization step. Then, it is important to "track" and correct continuously the CFO. The SCO can also be estimated and corrected if its drift is too important. For the developed software receiver a joint estimation of SCO and CFO using demodulated symbols is used. In theory, the delay has to be also tracked in order to have an FFT windows always in good position and avoid ISI. For this receiver, no specific function was developed for this purpose. As the position of the FFT window does not require high precision, the delay tracking can be done either by periodically redoing the CP synchronization, or by using the estimation of the delay made by the "navigation part" of the receiver presented in the next section.

Joint estimation of SCO and fractional CFO [6]: this algorithm requires computing the phase difference between the continuous pilot subcarriers of two consecutive OFDM symbols. According to (3), and assuming that the propagation channel is constant over two consecutive symbols $\left(H_{p}^{k}=\right.$ $H_{p}^{k+1}$ ), this phase difference is, for $p \in \mathcal{P}_{C}$ :

$$
\begin{aligned}
\theta_{p}^{k} & =\operatorname{angle}\left(\widetilde{d_{p}^{k+1}} \cdot{\widetilde{d_{p}^{k}}}^{*}\right) \\
& =\frac{2 \pi\left(N+N_{C P}\right)}{N}\left(\delta f+\delta_{S C O} p\right)+n
\end{aligned}
$$

Where $n$ is the noise term and $\mathcal{P}_{C}$ is the set of continuous subcarrier indexes:

Then $\delta f$ and $\delta_{S C O}$ can be estimated by performing a least square linear fitting of $\theta_{p}^{k}, p \in \mathcal{P}_{C}$. Thus:

$$
\begin{aligned}
& \hat{\delta}_{S C O}=\frac{N}{2 \pi\left(N+N_{C P}\right)} \frac{\overline{\boldsymbol{\theta p}}-\overline{\boldsymbol{\theta}} \cdot \overline{\boldsymbol{p}}}{\overline{\boldsymbol{p}}^{\mathbf{2}}-\overline{\boldsymbol{p}}^{\mathbf{2}}} \quad \text { and } \quad \widehat{\delta f}=\frac{N}{2 \pi\left(N+N_{C P}\right)}(\overline{\boldsymbol{\theta}}- \\
& \left.\hat{\delta}_{S C O} \overline{\boldsymbol{p}}\right)
\end{aligned}
$$

Where: $\overline{\boldsymbol{\theta} \boldsymbol{p}}=\sum_{p \in \mathcal{P}_{C}} \theta_{p}^{k} \cdot p, \overline{\boldsymbol{\theta}}=\sum_{p \in \mathcal{P}_{C}} \theta_{p}^{k}, \overline{\boldsymbol{p}}=\sum_{p \in \mathcal{P}_{C}} p$ and $\overline{\boldsymbol{p}^{\mathbf{2}}}=\sum_{p \in \mathcal{P}_{C}} p^{2}$

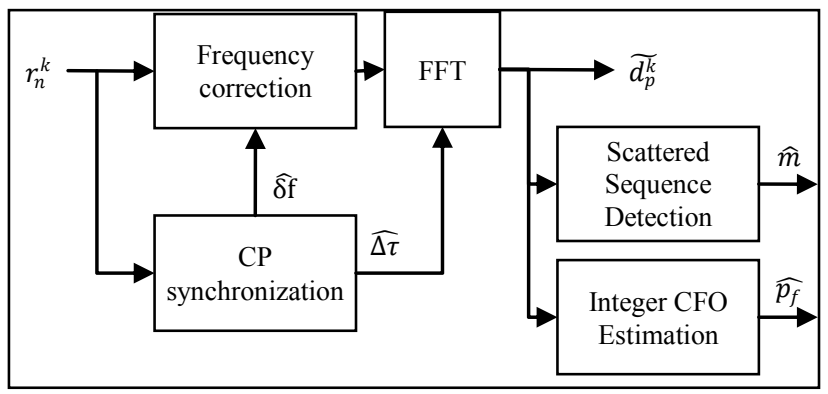

Figure 5. Initialization mode diagram 
To illustrate these equations, Fig. 6 shows the result of linear least square fitting with a residual $\mathrm{CFO}$ of 0.01 and a SCO of $100 \mathrm{ppm}$. In this example a DVB-T 2K simulated signal is used and the SNR is $20 \mathrm{~dB}$.

The estimated SCO and $\mathrm{CFO}$ are then corrected before the FFT. The CFO is corrected as in the acquisition mode and the $\mathrm{SCO}$ is corrected by oversampling and interpolating the received digital signal.

\section{Channel estimation and equalization [6]}

The goal of channel estimation and equalization is to cancel the distortions brought by the multipath propagation channel and residual synchronization errors and to recover the transmitted data symbols $d_{p}^{k}$. After correction of CFO, SCO and FFT window positioning the demodulated symbol is:

$$
\widetilde{d_{p}^{k}}=d_{p}^{k} \cdot \widetilde{H_{p}^{k}}+I C I+n_{p}^{k}
$$

Where:

- $\widetilde{H_{p}^{k}}=H_{p}^{k} \cdot \alpha\left(\varepsilon_{f}+\varepsilon_{S C O} p\right) \mathrm{e}^{j 2 \pi \frac{\varepsilon_{\tau} p}{N}} e^{j \phi_{k}\left(\varepsilon_{f}+\varepsilon_{S C O} p\right)}$

- $\varepsilon_{f}=\delta \mathrm{f}-\widehat{\delta f}$ the residual carrier frequency offset

- $\varepsilon_{S C O}=\delta_{S C O}-\hat{\delta}_{S C O}$ the residual sampling clock offset

- $\varepsilon_{\tau}=\tau-\hat{\tau}$ the residual timing offset

- $\quad$ ICI the ICI term due to residual CFO and SCO

- $n_{p}^{k}$ the noise term

$\widetilde{H_{p}^{k}}$ represents the complex coefficient that links the demodulated data symbol $\widetilde{d_{p}^{k}}$ and the transmitted one $d_{p}^{k}$. It includes the propagation channel term $H_{p}^{k}$ as well as the impact of all residual synchronization errors. The Least Square (LS) estimation of this complex coefficient on the pilot subcarriers (where the value of the transmitted symbol $d_{p}^{k}$ is known a priori), is:

$\widehat{H_{p}^{k}}=\frac{\widetilde{d_{p}^{k}}}{d_{p}^{k}}, p \in \mathcal{P}_{k}$ where $\mathcal{P}_{k}$ is the set of pilot subcarrier indexes for the k-th OFDM symbol.

Based on the values of $\widehat{H_{p}^{k}}$ for all the pilot subcarriers, the corresponding values for the data subcarriers can be easily obtained (through interpolation for instance). For a linear

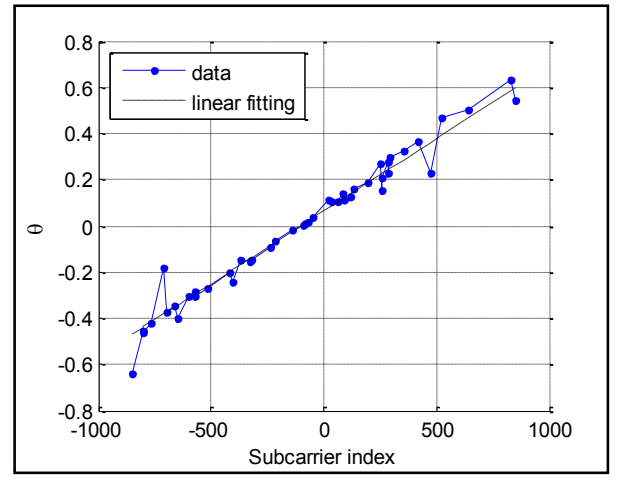

Figure 6. Joint $\mathrm{SCO}$ and $\mathrm{CFO}$ estimation interpolation:

$$
\widehat{H_{p}^{k}}=\left(1-\frac{p_{i}-p}{p_{i+1}-p_{i}}\right) \widehat{H}_{p_{i}}^{k}+\frac{p_{i+1}-p}{p_{i+1}-p_{i}} \widehat{H}_{p_{i+1}}^{k} \text { where } p_{i} \text { and } p_{i+1}
$$
are the pilot indexes around the data subcarrier index $p$.

Then the estimated transmitted symbols on the p-th subcarrier of the k-th OFDM symbol is:

$$
\widehat{d_{p}^{k}}=\frac{\widetilde{d_{p}^{k}}}{\widehat{H_{p}^{k}}}
$$

Fig. 7 shows the block diagram of the receiver in the operating mode. The estimated $\mathrm{CFO}$ and SCO are passed through a loop filter before correction in order to reduce estimation errors due to noise.

Supposing that the CFO and SCO are perfectly corrected, the Table II, derived the DVB-T standard [9], shows the required SNR at receiver input to have a Quasi Error Free (QEF) after decoding (less than one uncorrected error event per hour). This required SNR is given for the 3 possible data mapping constellations, for the different possible coding rate and for three types of channel (Gaussian channel, Ricean Channel, Rayleigh Channel). For the most adopted constellation, 16-QAM and 64-QAM, the required SNR is between $10 \mathrm{~dB}$ and $20 \mathrm{~dB}$.

\section{Structure of the NaVigation Part OF THE DEVELOPPED DVB-T SOFTWARE RECEIVER}

The navigation part of the developed software DVB-T receiver is derived from the algorithms detailed in [4]. It consists in obtaining precise synchronization by estimating the

TABLE II. REQUIRED SNR (IN DB) FOR QEF AFTER DECODING

\begin{tabular}{|c|c|c|c|c|}
\hline Modulation & $\begin{array}{c}\text { Code } \\
\text { rate }\end{array}$ & $\begin{array}{c}\text { Gaussian } \\
\text { Channel }\end{array}$ & $\begin{array}{c}\text { Ricean } \\
\text { Channel }\end{array}$ & $\begin{array}{c}\text { Rayleigh } \\
\text { Channel }\end{array}$ \\
\hline \multirow{4}{*}{ QPSK } & $1 / 2$ & 3.1 & 3.6 & 5.4 \\
\cline { 2 - 5 } & $2 / 3$ & 4.9 & 5.7 & 8.4 \\
\cline { 2 - 5 } & $3 / 4$ & 5.9 & 6.8 & 10.7 \\
\cline { 2 - 5 } & $5 / 6$ & 6.9 & 8.0 & 13.1 \\
\cline { 2 - 5 } & $7 / 8$ & 7.7 & 8.7 & 16.3 \\
\hline \multirow{4}{*}{ 16-QAM } & $1 / 2$ & 8.8 & 9.6 & 11.2 \\
\cline { 2 - 5 } & $2 / 3$ & 11.1 & 11.6 & 14.2 \\
\cline { 2 - 5 } & $3 / 4$ & 12.5 & 13.0 & 16.7 \\
\cline { 2 - 5 } & $5 / 6$ & 13.5 & 14.4 & 19.3 \\
\cline { 2 - 5 } & $7 / 8$ & 13.9 & 15.0 & 22.8 \\
\hline \multirow{5}{*}{ 64-QAM } & $1 / 2$ & 14.4 & 14.7 & 16.0 \\
\cline { 2 - 5 } & $2 / 3$ & 16.5 & 17.1 & 19.3 \\
\cline { 2 - 5 } & $3 / 4$ & 18.0 & 18.6 & 21.7 \\
\cline { 2 - 5 } & $5 / 6$ & 19.3 & 20.0 & 25.3 \\
\cline { 2 - 5 } & $7 / 8$ & 20.1 & 21.0 & 27.9 \\
\hline
\end{tabular}

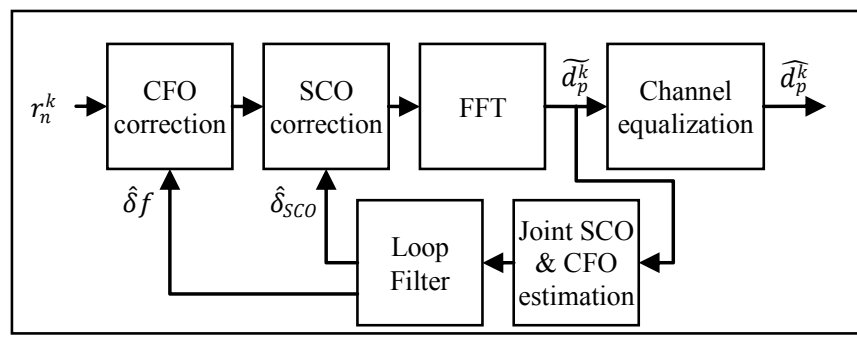

Figure 7. Operating mode diagram 
channel impulse response (CIR) thanks to the pilot subcarriers and with an Acquisition/Tracking strategy.

\section{A. Expression of the correlation function}

The CIR estimation is obtained by correlating the received signal with a local OFDM signal replica with only the scattered pilot subcarriers activated. Fig. 8 illustrates schematically this principle.

This correlation process is done in the frequency domain by multiplying the received pilot subcarriers by the locallygenerated pilot subcarriers (which are equal to the PRBS values) and then making an inverse Fourier transform (iDFT) of the result. Thus we have the following expression of the correlation function:

$$
R(\tau)=i D F T\left(\left\{\widetilde{d_{p}^{k}} P_{p}^{k^{*}}\right\}_{p \in \mathcal{P}_{S}(k)}\right)[\tau]
$$

Where:

- $\quad \tau$ is the correlation delay normalized by the sampling period

- $\quad R(\tau)$ is the value of the correlation function at $\tau$

- $\widetilde{d_{p}^{k}}$ is the demodulated symbol over the $\mathrm{p}$-th subcarrier of the k-th OFDM symbol

- $P_{p}^{k}$ is the value $(-1$ or +1$)$ of the pilot over the $\mathrm{p}$-th subcarrier of the k-th OFDM symbol

- $\mathcal{P}_{S}(k)$ is the set of scattered pilot indexes for the $\mathrm{k}$-th OFDM symbol

By developing the iDFT in (14) we obtain:

$$
R(\tau)=\frac{1}{\mathrm{~N}_{\mathrm{p}}} \sum_{p \in \mathcal{P}_{S}(k)} \widetilde{d_{p}^{k}} P_{p}^{k^{*}} \exp \left(j 2 \pi \frac{\tau p}{N}\right)
$$

With $N_{p}$ the number of scattered pilot subcarriers.

First, let's assume that only the useful signal is received with no noise, no multipath, no CFO and no SCO. Let also assume an attenuation $\alpha$ and a delay $\tau$ normalized by the sampling period $T_{\text {samp }}$ ) of the received signal, i.e. $r(t)=$ $\alpha s\left(t-\tau . T_{\text {samp }}\right)$. Then:

$$
R(\hat{\tau})=\frac{\alpha}{\mathrm{N}_{\mathrm{p}}} \sum_{p \in \mathcal{P}_{S}(k)} d_{p}^{k} P_{p}^{k^{*}} \exp \left(-\mathrm{j} 2 \pi \frac{\tau p}{N}\right) \exp \left(j 2 \pi \frac{\hat{\tau} p}{N}\right)
$$

As mentioned in section II.B, the pilot subcarriers of the DVB-T signals are BPSK modulated with a $4 / 3$ boosted amplitude, thus $d_{p}^{k} P_{p}^{k^{*}}=\frac{4}{3}$. In addition let $N_{0}$ be the number of Null subcarriers in the lower band of the OFDM signal

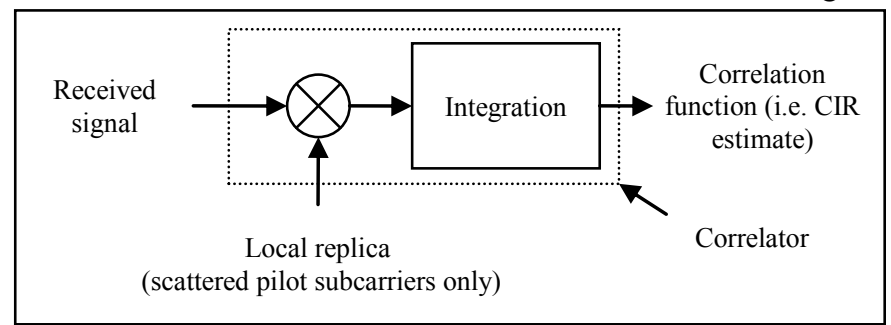

Figure 8. CIR estimation principle spectrum, $p_{0} \in\{3,6,9,12\}$ the index of the first scattered pilot and $P_{I}=12$ the scattered pilot interval (i.e. scattered pilots are inserted every 12 subcarriers). Then,

$R(\hat{\tau})=\frac{4}{3} \frac{\alpha}{N_{p}} \exp \left(j 2 \pi \frac{\left(N_{0}+p_{0}+\frac{P_{I}}{2}\left(N_{p}-1\right)\right)}{N} \varepsilon_{\tau}\right) \cdot \frac{\sin \left(\pi \frac{P_{I} \cdot N_{p}}{N} \varepsilon_{\tau}\right)}{\sin \left(\pi \frac{P_{I}}{N} \varepsilon_{\tau}\right)}$

with $\varepsilon_{\tau}=\hat{\tau}-\tau$. If $\varepsilon_{\tau}$ is small then the modulus of the correlation function becomes:

$$
|R(\hat{\tau})|=\frac{4}{3} \alpha \cdot\left|\operatorname{sinc}\left(\pi \beta \varepsilon_{\tau}\right)\right|
$$

Where $\operatorname{sinc}(x)=\sin (x) / x$ and $\beta=\frac{P_{I} N_{p}}{N}$ is a factor characterizing the width of the main lobe of the sinc function due to the presence of the null subcarriers. Fig. 9 confirms this formula by comparing the correlation function of a synthetic DVB-T signal with the expression of (18).

\section{B. Delay acquisition strategy}

As it was shown earlier, it is not necessary to have a very accurate timing synchronization to be able to demodulate the data message. Indeed, the residual timing error obtained after the $\mathrm{CP}$ synchronization of the communication part of the receiver presented in section III.A, is easily compensated by the equalization. However, to be able to initiate precise synchronization, as desired for navigation purpose, it is necessary to reach a finer delay acquisition.

Reference [4] proposes an adequate fine acquisition based on the matching pursuit algorithm [11]. It consists in decomposing the absolute value of the correlation function (which includes all the received signal replicas) in a sum of absolute sinc functions (thus representing the correlation function between one signal replica and the locally-generated signal). This decomposition provides an estimation of the delay and absolute amplitude of all the multipath components of the received signal with a finer accuracy than the CP synchronization algorithm. The drawback of this technique is that it uses the absolute value of the correlation function and is cumbersome. However, it showed to work sufficiently well for the present purpose.

\section{Delay Tracking strategy}

The delay tracking is realized using a classical Delay Lock Loop (DLL) structure. To do so, it was decided to use a normalized Early-Minus-Late Power (EMLP) discriminator. The expression of the normalized EMLP discriminator is:

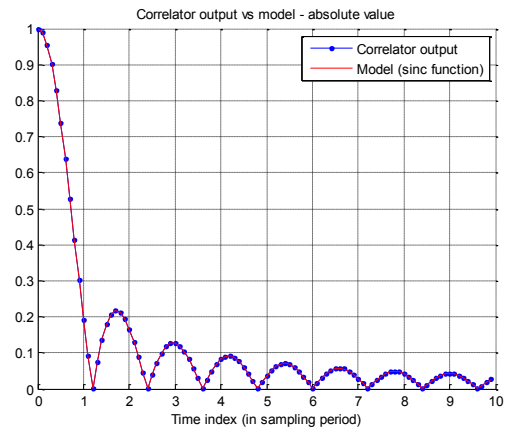

Figure 9. Correlator output vs model 


$$
D_{E M L P_{\text {norm }}}\left(\varepsilon_{\tau}\right)=\frac{\left|R\left(\hat{\imath}-\frac{\Delta}{2}\right)\right|^{2}-\left|R\left(\hat{\tau}+\frac{\Delta}{2}\right)\right|^{2}}{K_{\text {norm }}|R(\hat{\tau})|^{2}}
$$

Where $\Delta$ is the spacing in sample period between the Early and the Late correlator and $K_{\text {norm }}$ is a factor of normalization such that the discriminator has an unitary slope around $\varepsilon_{\tau}=0$. It can be shown that (see Appendix A for detailed calculation):

$$
K_{\text {norm }}=\frac{1-(\Delta / 2) \pi \beta \cdot \sin (\pi \beta \Delta)-\cos (\pi \beta \Delta)}{(\pi \beta)^{2} \cdot \Delta^{3} / 2^{4}}
$$

In addition, in order to have a discriminator output independent of the signal amplitude, a normalization by $|R(\hat{\tau})|^{2}$ is necessary. Thus, thanks to those normalizations, $D_{E M L P} P_{\text {norm }}\left(\varepsilon_{\tau}\right)=\varepsilon_{\tau}$ for $\varepsilon_{\tau}$ close to 0 as illustrated in Fig. 10.

The linearity zone of the discriminator is define as: $L=\arg \max _{\varepsilon_{\tau}}\left(\left|D_{E M L P_{\text {norm }}}\left(\varepsilon_{\tau}\right)-\varepsilon_{\tau}\right|<a\right)$

Fig. 11 illustrates the linearity zone of the normalized EMLP discriminator for $a=10 \%$. It shows that the linearity zone of the normalized EMLP discriminator slightly decreases when $\Delta$ augments, while remaining close to 0.5 samples. This is due to the shape of the correlation function presented in Fig. 9.

The EMLP discriminator is inserted in a DLL which architecture is shown in Fig. 12.

Thus, the value of the estimated delay $\hat{\tau}$ at the instant $k+1$ is the value of the estimated delay $\hat{\tau}$ at the instant $k$ corrected by the filtered value of the discriminator output $\widetilde{\varepsilon_{\tau}}$ at the instant $k$.

$$
\hat{\tau}^{k+1}=\hat{\tau}^{k}+{\widetilde{\varepsilon_{\tau}}}^{k}
$$

\section{Position computation strategy}

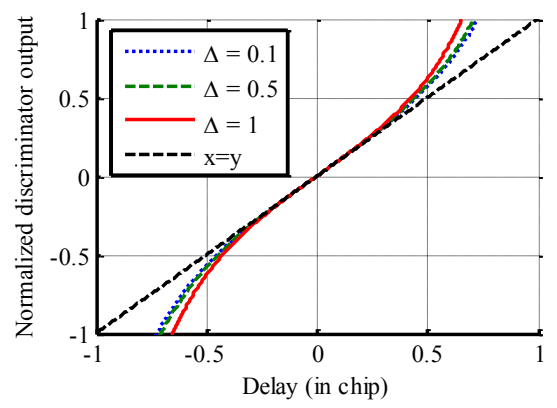

Figure 10. Normalized EMLP discriminator

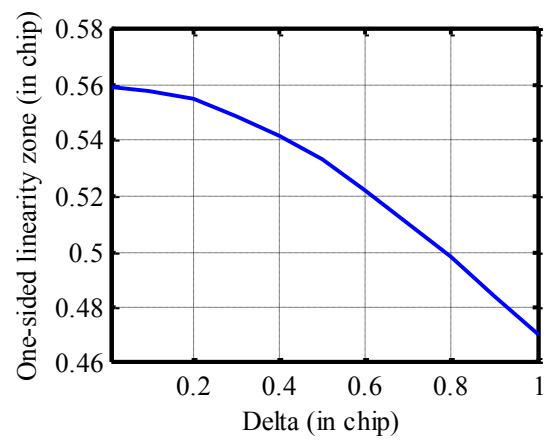

Figure 11. Linearity zone at $10 \%$ of the normalized EMLP discriminator
The position computation is not yet implemented in the software receiver, but different position computation scenarios can be defined depending on the type of network:

- In Single Frequency Networks (SFN) emitters share the same carrier frequency and are synchronized in time. Consequently, if the emitters' positions are known, a position computation is possible in theory, without external help, as in GNSS. There is however other issues, like transmitter discrimination (the correlation function has one peak per emitter) and identification (association of one peak with one emitter). Reference [12] proposes a method to handle those problems.

- In Multi-Frequency Networks (MFN) each emitter has its own carrier frequency and they are not synchronized. However, they generally use the same clock derived from GNSS. Thus, a position computation is impossible without a position reference, for instance an initial position obtained with a GNSS or by other mean. On the other hand, emitter discrimination and identification are not longer a problem since each emitter is identified by its carrier frequency.

For the two cases (SFN or MFN), an hybridization with GNSS is possible: the position computation module could use pseudorange measurements from both the DVB and GNSS receiver. Such hybridization would be more interesting in the MFN scenario where the number of emitters in sight, and therefore the number of pseudorange measurements from the DVB receiver, is usually more limited.

Fig. 13 summarizes this envisaged functioning of the future position computation module.

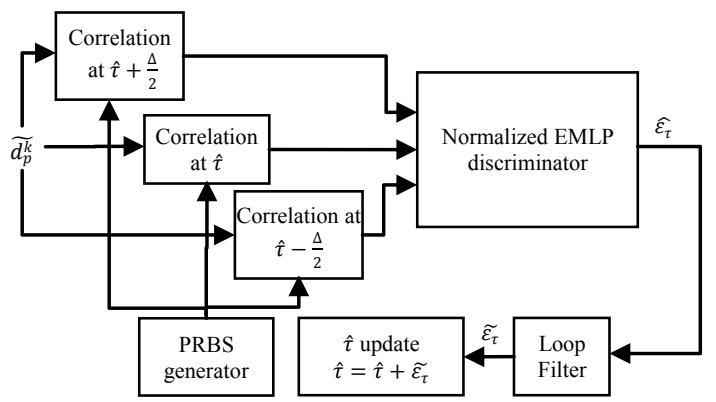

Figure 12. Tracking block diagram

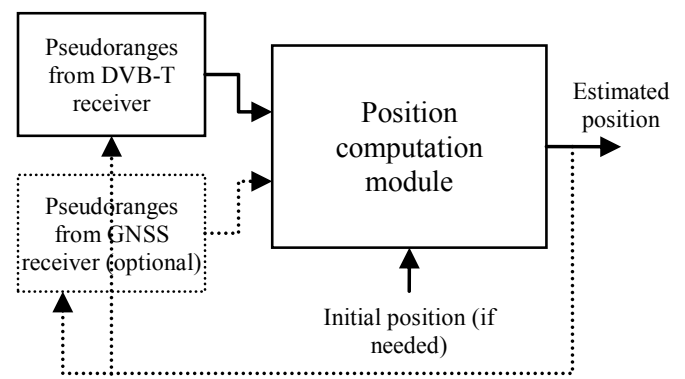

Figure 13. Possible position computation module 


\section{THEORETICAL AND SIMULATED PERFORMANCES OF} TRACKING

It is important to derive the theoretical expression of the performance of the proposed tracking algorithm for 2 reasons: (1) to validate the tracking concept, (2) to validate the receiver, and (3) to use this theoretical expression in order to correctly model the pseudorange measurements and their variance (for instance to test the performance of the positioning algorithm).

In the following, all simulations are done in a nominal DVB-T configuration: Mode $2 \mathrm{~K}, C P=\frac{1}{32}, T_{\text {samp }}=\frac{7}{64} \mu \mathrm{s}$

\section{A. Impact of noise on tracking}

To study the impact of noise on the tracking, all other impairments (CFO, SCO and multipath) are assumed negligible. Two criteria are studied in theory and by simulation: the variance of delay estimation and the tracking threshold.

1) Variance of the delay estimator

The discriminator output, supposing a tracking error $\varepsilon_{\tau}$, is:

$$
D_{E M L P_{\text {norm }}}\left(\varepsilon_{\tau}\right)=\frac{\left|R\left(\hat{\tau}-\frac{\Delta}{2}\right)\right|^{2}-\left|R\left(\hat{\tau}+\frac{\Delta}{2}\right)\right|^{2}}{K_{\text {norm }}|R(\hat{\tau})|^{2}}
$$

According to (16) and assuming that there is no multipath and only noise, the correlator output is:

$$
R(\hat{\tau})=R_{U}(\hat{\tau})+R_{N}(\hat{\tau})
$$

With:

- $R_{U}(\hat{\tau})=\frac{4}{3} \alpha \cdot \exp \left(j \Phi \varepsilon_{\tau}\right) \cdot \operatorname{sinc}\left(\pi \beta \varepsilon_{\tau}\right)$

- $\Phi=\pi \frac{\left(N_{0}+p_{0}+\frac{P_{I}}{2}\left(N_{p}-1\right)\right)}{N}$

- $\quad R_{N}(\hat{\tau})=\frac{1}{\mathrm{~N}_{\mathrm{p}}} \sum_{p \in \mathcal{P}_{S}(k)} n_{p}^{k} P_{p}^{k^{*}} \exp \left(j 2 \pi \frac{\hat{\tau} p}{N}\right)$

Then, by substituting (24) in (23) and after several manipulations, it can be deduced, for $\varepsilon_{\tau} \cong 0$ (see detailed calculations in Appendix B).

$$
\operatorname{Var}\left(D_{E M L P_{\text {norm }}}\right)=\frac{K_{1}}{S N R}\left[1+\frac{K_{2}}{S N R}\right]
$$

with :

$$
\begin{aligned}
\text { - } & K_{1}=\frac{9(1-\operatorname{sinc}(\pi \beta \Delta)) \operatorname{sinc}\left(\pi \beta \frac{\Delta}{2}\right)^{2}}{4 \cdot N_{P} \cdot K_{\text {norm }}{ }^{2}} \\
\text { - } \quad K_{2} & =\frac{9(1+\operatorname{sinc}(\pi \beta \Delta))}{32 N_{P} \operatorname{sinc}\left(\pi \beta \frac{\Delta}{2}\right)^{2}}
\end{aligned}
$$

To obtain this result it was assumed that the normalization was perfect. Of course this is not the completely the case in reality. However one can use instead of $|R(\hat{\tau})|^{2}$, an estimation of $\left|R_{U}(\hat{\tau})\right|^{2}$ by, for instance, averaging the noised value $|R(\hat{\tau})|^{2}$ over several iterations or by using any efficient signal power estimator.
This variance is normalized by the sampling period. Thus, the value of this variance converted in meter will increases when the sampling period increase. That is to say that the precision is better for the shortest sampling periods. In addition, this variance decrease when the number of pilot augments i.e. when the FFT size augment.

Fig. 14 shows a comparison between the simulated and theoretical standard deviations of the normalized EMLP discriminator output. In the calculus of the normalized EMLP discriminator output variance, it was supposed that $\varepsilon_{\tau} \cong 0$ and that the normalization was perfect. Thus, to validate this formula, the same assumptions were made in the simulation. Fig. 14 confirms the validity of (25).

In order to observe the influence of the early-late correlator spacing $\Delta$, Fig. 15 shows the theoretical standard deviation of the normalized EMLP for two values of $\Delta$. It clearly appears that the choice of $\Delta$ has only a limited influence on the tracking accuracy.

The next step is to calculate the variance of the estimated delay error, i.e. the output of the loop filter. The loop filter used is derived from [13]. Let $B_{L}$ be the bandwidth of the equivalent loop filter. The loop being updated every $T_{\text {symb }}$ second, where $T_{\text {symb }}=N(1+C P) T_{\text {samp }}$ denotes the length of an OFDM symbol, the variance at the output of the loop filter can be obtained using a result of [13]:

$$
\operatorname{Var}\left(\widetilde{\varepsilon_{\tau}}\right)=2 B_{L} T_{\text {symb }} \operatorname{Var}\left(D_{E M L P_{\text {norm }}}\left(\varepsilon_{\tau}\right)\right)
$$

Fig. 16 compares the theoretical and simulated standard deviations of the estimated delay. The loop bandwidth is fixed to $B_{L}=10 \mathrm{~Hz}$ and the OFDM symbol period is $231 \mu \mathrm{s}$.

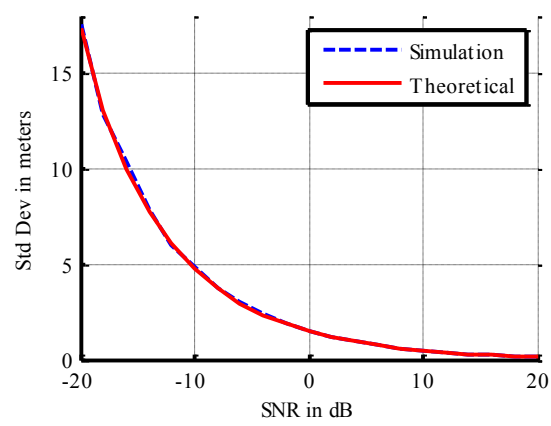

Figure 14. Simulated and Theoretical Standard Deviation of the normalized EMLP discriminator output

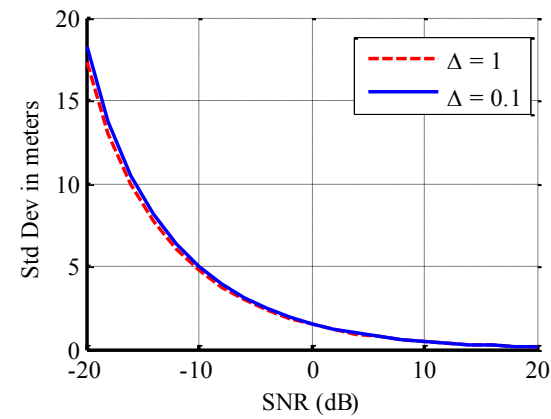

Figure 15. Influence of $\Delta$ on the theoretical standard deviation of the normalized EMLP 
Fig. 16 confirms the validity of (26). In addition on can notice that the pseudorange error standard deviation is under 2 meters for SNR greater than $-20 \mathrm{~dB}$ for the considered DVB-T signal configuration. Given that the required SNR for demodulation of the DTV signal is about 10-20 dB this tracking performance is excellent.

\section{2) Tracking Threshold}

The tracking threshold is the SNR threshold the loop can be considered to have lost tracking.

The classical condition of tracking loss is [13]:

$$
\text { 3. } \sigma_{\widetilde{\varepsilon_{\tau}}}<L
$$

where $L$ is the discriminator linearity zone defined in the section IV.C. Equation (27) can be rewritten as $\operatorname{Var}\left(\widetilde{\varepsilon_{\tau}}\right)<\frac{L^{2}}{9}$ and replacing $\operatorname{Var}\left(\widetilde{\varepsilon_{\tau}}\right)$ by its expression gives the following condition:

$$
2 B_{L} T_{\text {symb }} \frac{K_{1}}{S N R .}\left[1+\frac{K_{2}}{S N R}\right]<\frac{L^{2}}{9}
$$

The resolution of this quadratic inequality gives :

$$
S N R_{t h}=\frac{K+\sqrt{K^{2}+4 \cdot K \cdot K_{2}}}{2}
$$

With:

- $K=\frac{18 B_{L} T_{\text {symb }} K_{1}}{L^{2}}$

- $K_{1}=\frac{9(1-\operatorname{sinc}(\pi \beta \Delta)) \operatorname{sinc}\left(\pi \beta \frac{\Delta}{2}\right)^{2}}{4 \cdot N_{P} \cdot K_{\text {norm }}{ }^{2}}$

- $\quad K_{2}=\frac{9(1+\operatorname{sinc}(\pi \beta \Delta))}{32 N_{P} \operatorname{sinc}\left(\pi \beta \frac{\Delta}{2}\right)^{2}}$

To validate the expression of the tracking threshold, the 3sigma value of the loop filter output is computed over 5000 OFDM symbols. This operation is done for several values of SNR and several values of Early-Late correlators spacing $\Delta$.

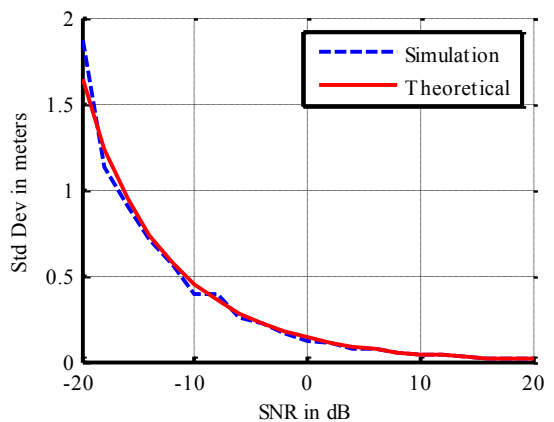

Figure 16. Simulated and Theoretical Standard Deviation of the loop filter output
For each value of $\Delta$, the SNR from which the 3-sigma value of the loop filter output is below the linearity zone $L$, gives the simulated tracking threshold. For this simulation, $B_{L}=10 \mathrm{~Hz}$ This simulated threshold is compared to the theoretical one on Fig. 17.

For the second simulation $\Delta$ is fixed at 1 and $B_{L}$ varies. The simulated threshold obtained is compared to the theoretical one on Fig. 18.

On Fig. 17 and Fig. 18 the closeness of theoretical and simulated results validates the expression of tracking threshold. Fig. 17 shows that the tracking threshold is almost independent of the value of $\Delta$. As expected, Fig 18 shows that increasing the value of the equivalent loop bandwidth (i.e. less filtering of the noise) increases the tracking threshold.

Tracking threshold values lower than $-25 \mathrm{~dB}$ are observed, which is very good compared to the required SNR for demodulation of TV signal around 10-20 dB. This margin could permit to track the TV signal in environments where the data demodulation will not be possible but where the SNR will still be above the tracking threshold, for instance in deep indoor. This provides a great hope to be able to process signals coming from several emitters, even indoors.

\section{B. Impact of multipath on tracking}

To observe the influence of multipath on delay estimation, the multipath error envelop is represented on Fig. 19 for several values of the Early-Late correlators spacing $\Delta$. This representation, typical in GNSS, shows the maximum negative and positive errors on delay estimation with respect to the delay of one multipath of amplitude equals to 0.5 relatively to the amplitude of the LOS signal. In this simulation of DVB-T $2 \mathrm{~K}$ signal is used.

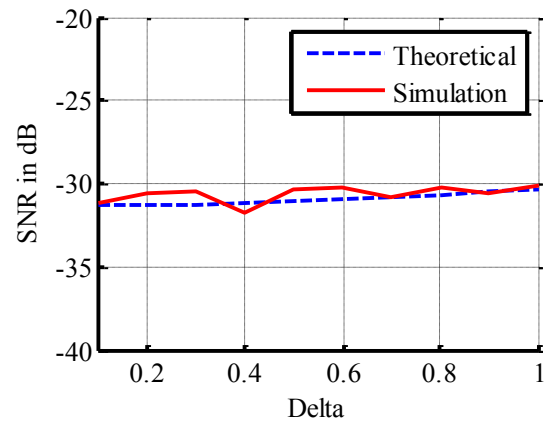

Figure 17. Simulated and theoretical tracking threshold for different values of early-late correlators spacing

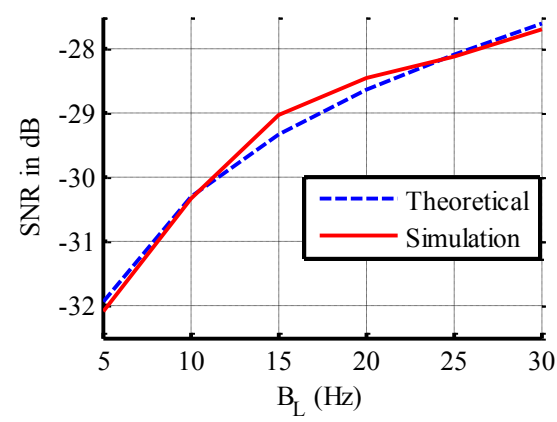

Figure 18. Simulated and theoretical tracking threshold for different $B_{L}$ 


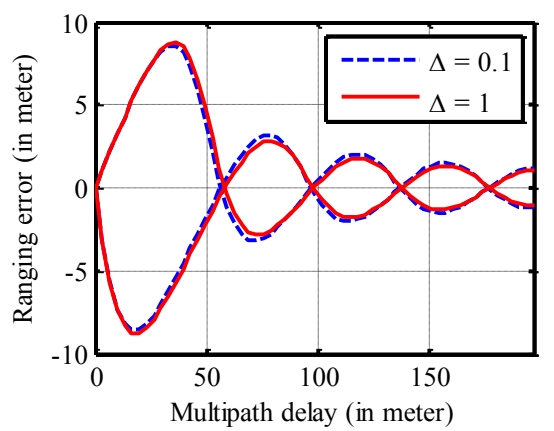

Figure 19. Multipath error envelop

It can be seen that the multipath error envelop is almost independent of the Early-Late correlators spacing $\Delta$. In addition, one can observe that even long multipath delays produce errors on the delay estimation. This is due to oscillation of the correlation function (sinus cardinal). This means that strong multipath, even if they are significantly delayed, will impact the timing tracking. This is a clear drawback when considering that the relative amplitude of the different received signals can be quite high in constrained environment. This problem will likely have to be tackled specifically.

\section{PERFORMANCE IN A REALISTIC CHANNEL}

Results presented in the previous section suppose a LOS channel without multipath. If this hypothesis could be valid for satellite transmission, it is completely hazardous for terrestrial transmission, considered in this article. Even, in most of the considered urban environment or indoor, the LOS signal is absent. This configuration is known as Non Line-Of-Sight (NLOS) transmissions.

Even for multipath error envelop study, the representation (LOS +one multipath of half amplitude) is not well adapted for terrestrial communications where multipath signals are numerous and potentially more powerful than LOS signal. Consequently, it is important to consider a more realistic multipath channel adapted to urban propagation.

One of the solutions is to use the validation channel models proposed in the DVB-T standard [9]. The standard introduces two models: a Rice channel model and a Rayleigh channel model. They are both constituted of 20 multipath (plus a LOS tap for the Rice channel) . Fig. 20 shows the channel impulse response (CIR) (multipath delays and associated average amplitudes) of this model. The specified

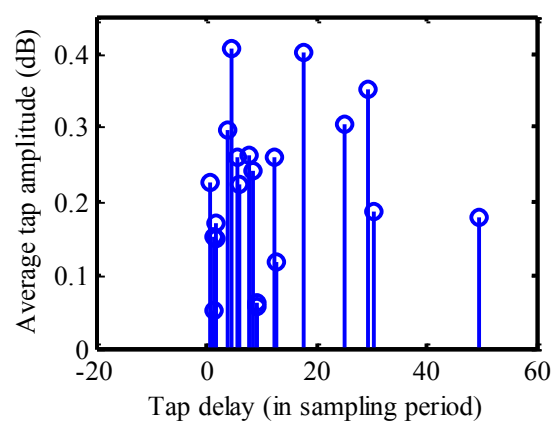

Figure 20. Tap delays and amplitudes of DVB-T channel model amplitudes are the mean values of the generated real tap amplitudes. The generated tap amplitudes are time-varying according to a Rayleigh distribution and their variation depends on the speed of the receiver.

Fig. 21 shows the correlation function obtained with a synthetic DVB-T $2 \mathrm{~K}$ signal passed through the channel impulse response generated according the tap configuration of Fig. 20.

The delay acquisition is achieved by taking the delay corresponding to the maximum of the correlation function of Fig. 21. This delay is used to initialize the tracking loop. Fig. 22 shows the pseudorange error obtained. In this simulation a movement of the receiver at $5 \mathrm{~m} \cdot \mathrm{s}^{-1}$ is supposed, a DVB-T $2 \mathrm{~K}$ synthetic signal is used, the bandwidth of the equivalent tracking loop is set at $5 \mathrm{~Hz}$ and the early-late correlator spacing is 1 sample. The SNR is $10 \mathrm{~dB}$.

In this example the RMSE is about 10 meters. The variations around the real value are the consequences of the variations of tap amplitude due to movement of the receiver and the time response of the loop.

Even in multipath channel the tracking performance seems to be quite good. However, it is important to note that the aim of this result is only to show the behavior of the tracking in a urban channel. Indeed, this result corresponds to the tracking error of a particular peak of the correlation function which can be a multipath. Tracking a multipath induces a bias in the pseudorange error corresponding to the distance between the LOS and this multipath which can be very important. Thus, solutions to mitigate this bias has to be found in order to propose a complete positioning service. A solution to reduce this bias due to the tracking of a multipath has already been proposed in [4].

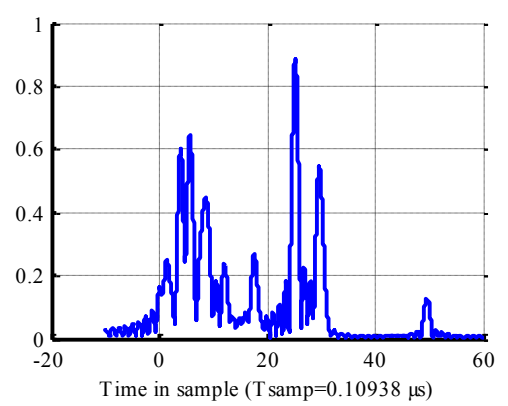

Figure 21. Correlation function in multipath channel.

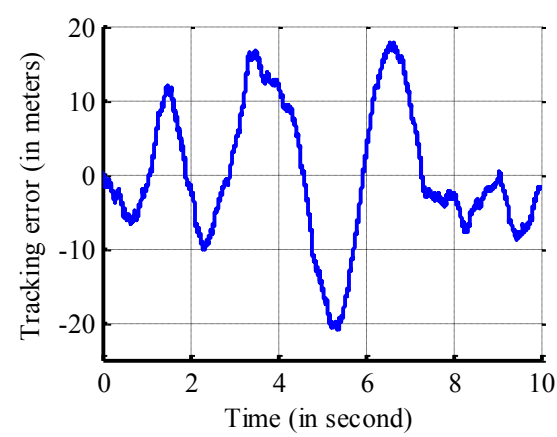

Figure 22. Pseudorange estimation error 
In addition, the multipath channel model uses fixed tap delays. In this model only the tap amplitude is varying. If such a model is totally adequate for telecommunication, it can however be considered limited for a navigation purpose, where the tap delay should be related to the movement of the sensor.

\section{CONCLUSION AND FUTURE WORK}

This article proposes a method to extract pseudorange measurement from the DVB-T DTV signal based on OFDM modulation. The first theoretical expressions of variance of pseudorange error and tracking threshold are given in case of LOS signal. These expressions are validated by simulation using the developed DVB-T software receiver. The pseudorange error sigma is around a few meters and the tracking threshold is below $-20 \mathrm{~dB}$.

The tracking performance was studied in a more realistic channel. The RMSE of delay estimation is about 10 meters for the particular studied example, which is quite good considering the time-varying channel. However the used channel model, using fix tap delays, is not totally satisfying for a navigation purpose and complementary studies on real signal have to be done to confirm these good results.

In addition, solutions to solve the multipath peak tracking issue have to be found in order to provide a complete positioning service.

\section{REFERENCE}

[1] "Rosum FemtoSynch In-Building Timing and Location for Femtocells", www.rosum.com/Rosum FemtoSynch White Paper_Oct08.pdf. Rosum Corporation, $200 \overline{8}$.

[2] "SKYHOOK Wireless.", www.skyhookwireless.com/howitworks/

[3] "DVB - Digital Video Broadcasting - DVB Worldwide." http://www.dvb.org/about_dvb/dvb_worldwide/

[4] P. Thevenon, O. Julien, C. Macabiau, D. Serant, S. Corazza, M. Bousquet, L. Ries, et T. Grelier, "Pseudo-Range Measurements Using OFDM Channel Estimation," ION GNSS 2009, 2009.

[5] J.A. Bingham, "Multicarrier modulation for data transmission: An idea whose time has come," IEEE Communications magazine, vol. 28, 1990, p. 5-14.

[6] T.D. Chiueh et P. Tsai, OFDM Baseband Receiver Design for Wireless Communications, John Wiley \& Sons (Asia) Pte Ltd, 2007.

[7] T. Pollet, P. Spruyt, et M. Moeneclaey, "The BER performance of OFDM systems using non-synchronized sampling," Global Telecommunications Conference, 1994. GLOBECOM '94. Communications: The Global Bridge., IEEE, 1994, p. 257 vol.1, 253.

[8] M. Morelli, C. Kuo, et M. Pun, "Synchronization Techniques for Orthogonal Frequency Division Multiple Access (OFDMA): A Tutorial Review," Proceedings of the IEEE, vol. 95, 2007, p. 1427, 1394.

[9] "Digital Video Broadcasting (DVB); Framing structure, channel coding and modulation for digital terrestrial television," Juin. 2008.

[10] J. van de Beek, M. Sandell, et P. Borjesson, "ML estimation of time and frequency offset in OFDM systems," Signal Processing, IEEE Transactions on [see also Acoustics, Speech, and Signal Processing, IEEE Transactions on], vol. 45, 1997, p. 1805, 1800.

[11] S.F. Cotter et B.D. Rao, "Sparse channel estimation via matching pursuit with application to equalization," IEEE Transactions on Communications, vol. 50, 2002, p. 374-377.

[12] P. Thevenon, O. Julien, C. Macabiau, D. Serant, L. Ries, S. Corazza, et M. Bousquet, "Positioning principles with a mobile TV system using DVB-SH signals and a Single Frequency Network," Digital Signal Processing, 2009 16th International Conference on, 2009, p. 8, 1.

[13] E. Kaplan et C. Hegarty, Understanding GPS: Principles And Applications, Artech House, 2005.

[14] L. Isserlis, "On a formula for the product-moment coefficient of any order of a normal frequency distribution in any number of variables," Biometrika, vol. 12, 1918, p. 134.

\section{APPENDIX A}

\section{Calculation of the coefficient of normalization}

The goal of normalization is that the discriminator has an unitary slope around $\varepsilon_{\tau}=0$. To this end, let us find an asymptotic equivalent of the non-normalized discriminator around $\varepsilon_{\tau}=0$.

Forgetting the constant coefficients this discriminator is:

$$
\begin{aligned}
& D\left(\varepsilon_{\tau}\right)=\frac{\sin \left(\pi \beta\left(\varepsilon_{\tau}-\Delta / 2\right)\right)^{2}}{(\pi \beta)^{2}\left(\varepsilon_{\tau}-\Delta / 2\right)^{2}}-\frac{\sin \left(\pi \beta\left(\varepsilon_{\tau}+\Delta / 2\right)\right)^{2}}{(\pi \beta)^{2}\left(\varepsilon_{\tau}+\Delta / 2\right)^{2}} \\
& D\left(\varepsilon_{\tau}\right)=\frac{\sin \left(\pi \beta\left(\varepsilon_{\tau}-\Delta / 2\right)\right)^{2} \cdot\left(\varepsilon_{\tau}+\Delta / 2\right)^{2}-\sin \left(\pi \beta\left(\varepsilon_{\tau}+\Delta / 2\right)\right)^{2} \cdot\left(\varepsilon_{\tau}-\Delta / 2\right)^{2}}{(\pi \beta)^{2} \cdot\left(\varepsilon_{\tau}-\Delta / 2\right)^{2} \cdot\left(\varepsilon_{\tau}+\Delta / 2\right)^{2}} \\
& D\left(\varepsilon_{\tau}\right)=\frac{\frac{1-\cos \left(\pi \beta\left(2 \cdot \varepsilon_{\tau}-\Delta\right)\right)}{2} \cdot\left(\varepsilon_{\tau}+\Delta / 2\right)^{2}-\frac{1-\cos \left(\pi \beta\left(2 \cdot \varepsilon_{\tau}+\Delta\right)\right)}{2} \cdot\left(\varepsilon_{\tau}-\Delta / 2\right)^{2}}{(\pi \beta)^{2} \cdot\left(\varepsilon_{\tau}-\Delta / 2\right)^{2} \cdot\left(\varepsilon_{\tau}+\Delta / 2\right)^{2}} \\
& D\left(\varepsilon_{\tau}\right)=\frac{\frac{1-\cos \left(\pi \beta\left(2 \cdot \varepsilon_{\tau}-\Delta\right)\right)}{2} \cdot\left(\varepsilon_{\tau}+\Delta / 2\right)^{2}-\frac{1-\cos \left(\pi \beta\left(2 \cdot \varepsilon_{\tau}+\Delta\right)\right)}{2} \cdot\left(\varepsilon_{\tau}-\Delta / 2\right)^{2}}{(\pi \beta)^{2} \cdot\left(\varepsilon_{\tau}-\Delta / 2\right)^{2} \cdot\left(\varepsilon_{\tau}+\Delta / 2\right)^{2}}
\end{aligned}
$$$$
D\left(\varepsilon_{\tau}\right)=\left\{\begin{array}{l}
\frac{1}{(\pi \beta)^{2} \cdot\left(\varepsilon_{\tau}-\Delta / 2\right)^{2} \cdot\left(\varepsilon_{\tau}+\Delta / 2\right)^{2}}\left[\frac{\left(\varepsilon_{\tau}+\Delta / 2\right)^{2}-\left(\varepsilon_{\tau}+\Delta / 2\right)^{2}}{2}\right. \\
+\frac{\left(\varepsilon_{\tau}^{2}+(\Delta / 2)^{2}\right)\left(\cos \left(\pi \beta\left(2 . \varepsilon_{\tau}+\Delta\right)\right)-\cos \left(\pi \beta\left(2 . \varepsilon_{\tau}-\Delta\right)\right)\right.}{2} \\
\left.-\frac{\varepsilon_{\tau} \cdot \Delta\left(\cos \left(\pi \beta\left(2 \varepsilon_{\tau}+\Delta\right)\right)+\cos \left(\pi \beta\left(2 . \varepsilon_{\tau}-\Delta\right)\right)\right.}{2}\right]
\end{array}\right.
$$

$D\left(\varepsilon_{\tau}\right)=$

$\frac{\varepsilon_{\tau} \cdot \Delta-\left(\varepsilon_{\tau}{ }^{2}+(\Delta / 2)^{2}\right) \sin \left(2 \pi \beta \varepsilon_{\tau}\right) \sin (\pi \beta \Delta)-\varepsilon_{\tau} \cdot \Delta \cdot \cos \left(2 \pi \beta \varepsilon_{\tau}\right) \cos (\pi \beta \Delta)}{(\pi \beta)^{2} \cdot\left(\varepsilon_{\tau}-\Delta / 2\right)^{2} \cdot\left(\varepsilon_{\tau}+\Delta / 2\right)^{2}}$

Thus,

$$
\frac{D\left(\varepsilon_{\tau}\right)}{\varepsilon_{\tau}} \underset{\varepsilon_{\tau} \rightarrow 0}{\longrightarrow} \frac{1-(\Delta / 2) \pi \beta \cdot \sin (\pi \beta \Delta)-\cos (\pi \beta \Delta)}{(\pi \beta)^{2} \cdot \Delta^{3} / 2^{4}} \stackrel{\text { def }}{=} K_{\text {norm }}
$$

\section{APPENDIX B}

\section{Calculation of the variance of the discriminator output}

The noisy correlation is considered:

$$
R(\hat{\tau})=R_{U}(\hat{\tau})+R_{N}(\hat{\tau})
$$

As preliminary results, the statistics of the noise term of the correlation are:

$$
\begin{aligned}
& E\left[R_{N}(\hat{\tau})\right]=0 \text { and, } \\
& \operatorname{Var}\left(R_{N}(\hat{\tau})\right)=E\left[R_{N}(\hat{\tau}) R_{N}(\hat{\tau})^{*}\right]=\frac{\sigma_{n}^{2}}{N_{P}} \text { where } \sigma_{n}^{2} \text { is the }
\end{aligned}
$$
variance of the noise on the demodulated subcarriers.

The non-normalized discriminator output is:

$$
D\left(\varepsilon_{\tau}\right)=\left|R\left(\hat{\imath}-\frac{\Delta}{2}\right)\right|^{2}-\left|R\left(\hat{\imath}+\frac{\Delta}{2}\right)\right|^{2}
$$

But:

$$
\begin{aligned}
\left|R\left(\hat{\tau}-\frac{\Delta}{2}\right)\right|^{2} & =R\left(\hat{\tau}-\frac{\Delta}{2}\right) R\left(\hat{\tau}-\frac{\Delta}{2}\right)^{*} \text { Let us express each } \\
& =\left|R_{U}\left(\hat{\tau}-\frac{\Delta}{2}\right)\right|^{2}+N^{-}
\end{aligned}
$$

term of the discriminator: 


$$
\begin{aligned}
\left|R\left(\hat{\tau}-\frac{\Delta}{2}\right)\right|^{2} & =R\left(\hat{\tau}-\frac{\Delta}{2}\right) R\left(\hat{\tau}-\frac{\Delta}{2}\right)^{*} \\
& =\left|R_{U}\left(\hat{\tau}-\frac{\Delta}{2}\right)\right|^{2}+N^{-}
\end{aligned}
$$

with:

$$
\begin{aligned}
& N^{-}=N\left(\hat{\tau}-\frac{\Delta}{2}\right) \text { and, } \\
& N(\hat{\tau})=R_{U}\left(\varepsilon_{\tau}\right) R_{N}(\hat{\tau})^{*}+R_{N}(\hat{\tau}) R_{U}\left(\varepsilon_{\tau}\right)^{*}+\left|R_{N}(\hat{\tau})\right|^{2}
\end{aligned}
$$
In the same way:

$$
\left|R\left(\hat{\imath}+\frac{\Delta}{2}\right)\right|^{2}=\left|R_{U}\left(\hat{\imath}+\frac{\Delta}{2}\right)\right|^{2}+N^{+}
$$

with:

$$
N^{+}=N\left(\hat{\imath}+\frac{\Delta}{2}\right)
$$

Equivalently, let us introduce the following notation:

$$
\begin{aligned}
& R_{U}^{-}=R_{U}\left(\hat{\imath}-\frac{\Delta}{2}\right), R_{U}^{+}=R_{U}\left(\hat{\imath}+\frac{\Delta}{2}\right) \text { and, } \\
& R_{N}^{-}=R_{N}\left(\hat{\imath}-\frac{\Delta}{2}\right), R_{N}^{+}=R_{N}\left(\hat{\imath}+\frac{\Delta}{2}\right)
\end{aligned}
$$

Thus the expression of non-normalized discriminator can be rewritten as:

$$
D\left(\varepsilon_{\tau}\right)=\left|R_{U}^{-}\right|^{2}-\left|R_{U}^{+}\right|^{2}+\mathcal{N}
$$

with $\mathcal{N}=N^{-}-N^{+}$the global noise of the discriminator.

We look at the statistics of this noise. First, its mean:

$$
E[\mathcal{N}]=E\left[N^{-}\right]-E\left[N^{+}\right]=\frac{\sigma_{n}^{2}}{N_{P}}-\frac{\sigma_{n}^{2}}{N_{P}}=0
$$

Secondly, the calculus of its variance is a little more complex:

$$
\operatorname{Var}(\mathcal{N})=E\left[\mathcal{N} \mathcal{N}^{*}\right]
$$

But:

$$
\begin{array}{rlc}
\mathcal{N} \mathcal{N}^{*} & =\left(N^{-}-N^{+}\right)\left(N^{-}-N^{+}\right)^{*} \\
& =\left|N^{-}\right|^{2}+\left|N^{+}\right|^{2}-2 \cdot \operatorname{Re}\left(N^{-} \cdot N^{+*}\right)
\end{array}
$$

Thus, the expectation of three terms has to be calculated:

First, $E\left[\left|N^{-}\right|^{2}\right]$ :

$$
\begin{aligned}
\left|N^{-}\right|^{2}= & N^{-} N^{-*} \\
= & \left(R_{U}^{-} R_{N}^{-*}+R_{N}^{-} R_{U}^{-*}+\left|R_{N}^{-}\right|^{2}\right) \times \\
& \left(R_{U}^{-} R_{N}^{-*}+R_{N}^{-} R_{U}^{{ }^{*}}+\left|R_{N}^{-}\right|^{2}\right)^{*}
\end{aligned}
$$

As $R_{N}$ is a complex normal variable the following properties of complex normal random variable can be used:

$E\left[R_{N}^{-} R_{N}^{-}\right]=0$

$E\left[R_{N}^{-(*)} R_{N}^{-(*)} R_{N}^{-(*)}\right]=0$ (the 3 rd order moment of a complex normal variable is null).

Thus:

$$
\begin{aligned}
E\left[\left|N^{-}\right|^{2}\right] & =2\left|R_{U}^{-}\right|^{2} E\left[\left|R_{N}^{-}\right|^{2}\right]+E\left[\left|R_{N}^{-}\right|^{4}\right] \\
& =2\left|R_{U}^{-}\right|^{2} \frac{\sigma_{n}^{2}}{N_{P}}+2 \frac{\sigma_{N}^{4}}{N_{P}^{2}}
\end{aligned}
$$

Secondly, $E\left[\left|N^{+}\right|^{2}\right]$ :

In the same way than the previous calculation:

$$
E\left[\left|N^{+}\right|^{2}\right]=2\left|R_{U}^{+}\right|^{2} \frac{\sigma_{n}^{2}}{N_{P}}+2 \frac{\sigma_{N}^{4}}{N_{P}^{2}}
$$

Thirdly, $E\left[N^{-} \cdot N^{+*}\right]$ :

$$
\begin{aligned}
N^{-} \cdot N^{+^{*}}= & \left(R_{U}^{-} R_{N}^{-*}+R_{N}^{-} R_{U}^{-*}+\left|R_{N}^{-}\right|^{2}\right) \times \\
& \left(R_{U}^{+} R_{N}^{+*}+R_{N}^{+} R_{U}^{+*}+\left|R_{N}^{+}\right|^{2}\right)^{*}
\end{aligned}
$$

Using the same properties of normal variables than previously, it comes:

$$
\begin{aligned}
E\left[N^{-} . N^{+*}\right]= & R_{U}^{-} R_{U}^{+*} E\left[R_{N}^{-*} R_{N}^{+}\right]+ \\
& R_{U}^{-*} R_{U}^{+} E\left[R_{N}^{-} R_{N}^{+*}\right]+ \\
& E\left[\left|R_{N}^{-}\right|^{2}\left|R_{N}^{+}\right|^{2}\right]
\end{aligned}
$$

One can notice, from the expression of $R_{N}(\hat{\tau})$ (see (24)), that: $E\left[R_{N}^{-*} R_{N}^{+}\right]=e^{j \Phi \Delta} \operatorname{sinc}(\pi \beta \Delta) \frac{\sigma_{n}^{2}}{N_{P}}$,

$E\left[R_{N}^{-} R_{N}^{+*}\right]=e^{-j \Phi \Delta} \operatorname{sinc}(\pi \beta \Delta) \frac{\sigma_{n}^{2}}{N_{P}}$,

$R_{U}^{-} R_{U}^{+*}=A^{-} A^{+} . e^{-j \Phi \Delta}$ and,

$R_{N}^{-*} R_{N}^{\mp}=A^{-} A^{+} \cdot e^{j \Phi \Delta}$.

where $A^{-}=A\left(\varepsilon_{\tau}-\frac{\Delta}{2}\right)$,

$$
\begin{aligned}
& A^{+}=A\left(\varepsilon_{\tau}+\frac{\Delta}{2}\right) \text { and, } \\
& A\left(\varepsilon_{\tau}\right)=\frac{4}{3} \alpha \operatorname{sinc}\left(\pi \beta \varepsilon_{\tau}\right)
\end{aligned}
$$

In addition using this property of 4 normal random variables $N_{1}, N_{2}, N_{3}, N_{4}$ (Isserlis' theorem [14]):

$$
\begin{aligned}
E\left[N_{1} N_{2} N_{3} N_{4}\right]= & E\left[N_{1} N_{2}\right] E\left[N_{3} N_{4}\right]+E\left[N_{1} N_{3}\right] E\left[N_{2} N_{4}\right]+ \\
& E\left[N_{1} N_{4}\right] E\left[N_{2} N_{3}\right]
\end{aligned}
$$

It comes:

$$
\begin{aligned}
E\left[\left|R_{N}^{-}\right|^{2}\left|R_{N}^{+}\right|^{2}\right]= & E\left[\left|R_{N}^{-}\right|^{2}\right] E\left[\left|R_{N}^{+}\right|^{2}\right]+ \\
& E\left[R_{N}^{-*} R_{N}^{+}\right] E\left[R_{N}^{-} R_{N}^{+*}\right]+ \\
& E\left[R_{N}^{-} R_{N}^{+}\right] E\left[R_{N}^{-*} R_{N}^{+*}\right] \\
= & \frac{\sigma_{n}^{4}}{N_{P}^{2}}\left(1+\operatorname{sinc}(\pi \beta \Delta)^{2}\right)
\end{aligned}
$$

Thus,

$E\left[N^{-} N^{+*}\right]=2 A^{-} A^{+} \operatorname{sinc}(\pi \beta \Delta) \frac{\sigma_{n}^{2}}{N_{P}}+\left(1+\operatorname{sinc}(\pi \beta \Delta)^{2}\right) \frac{\sigma_{n}^{4}}{N_{P}^{2}}$

Finally:

$$
\begin{aligned}
& \operatorname{Var}(\mathcal{N})= E\left[\left|N^{-}\right|^{2}+\left|N^{+}\right|^{2}-2 \cdot \operatorname{Re}\left(N^{-} \cdot N^{+*}\right)\right] \\
&= 2\left|R_{U}^{-}\right|^{2} \frac{\sigma_{n}^{2}}{N_{P}}+2 \frac{\sigma_{N}^{4}}{N_{P}^{2}}+ \\
& 2\left|R_{U}^{+}\right|^{2} \frac{\sigma_{n}^{2}}{N_{P}}+2 \frac{\sigma_{N}^{4}}{N_{P}^{2}}- \\
& 2\left(2 A^{-} A^{+} \operatorname{sinc}(\pi \beta \Delta) \frac{\sigma_{n}^{2}}{N_{P}}+\left(1+\operatorname{sinc}(\pi \beta \Delta)^{2}\right) \frac{\sigma_{n}^{4}}{N_{P}^{2}}\right) \\
&= 2 \frac{\sigma_{n}^{2}}{N_{P}}\left[\left|R_{U}^{-}\right|^{2}+\left|R_{U}^{+}\right|^{2}-2 A^{-} A^{+} \operatorname{sinc}(\pi \beta \Delta)+\right. \\
&\left.\frac{\sigma_{n}^{2}}{N_{P}}\left(1-\operatorname{sinc}(\pi \beta \Delta)^{2}\right)\right]
\end{aligned}
$$

Let us consider the normalization of the DLL:

$$
\operatorname{Var}\left(D_{\text {norm }}\left(\varepsilon_{\tau}\right)\right)=\operatorname{Var}\left(\frac{D\left(\varepsilon_{\tau}\right)}{K_{\text {norm }}\left|R_{U}\right|^{2}}\right)=\frac{\operatorname{Var}\left(D\left(\varepsilon_{\tau}\right)\right)}{K_{\text {norm }}^{2}\left|R_{U}\right|^{4}}
$$

Supposing that the tracking is good (SNR condition are favorable), then it can be supposed that $\varepsilon_{\tau} \approx 0$. In addition, the SNR is define as the ratio between the power of the data subcarriers and the power of noise:

$$
\operatorname{SNR}=\frac{\alpha^{2}}{\sigma_{n}^{2}}
$$


Thus, under the condition $\varepsilon_{\tau} \approx 0$, and keeping in mind that $\left|R_{U}\right|^{2}=\frac{16}{9} \alpha^{2} \operatorname{sinc}\left(\pi \beta \varepsilon_{\tau}\right)^{2}$, it comes:

$$
\begin{aligned}
\operatorname{Var}\left(D_{\text {norm }}\left(\varepsilon_{\tau}\right)\right)= & \frac{9}{16} \frac{2}{N_{P} K_{\text {norm }} \mathrm{SNR}} \times \\
& {\left[2 \operatorname{sinc}\left(\pi \beta \frac{\Delta}{2}\right)^{2}(1-\operatorname{sinc}(\pi \beta \Delta))+\right.} \\
& \left.\frac{9}{16} \frac{1}{N_{P} S N R}\left(1-\operatorname{sinc}(\pi \beta \Delta)^{2}\right)\right]
\end{aligned}
$$

Thus

with:

$$
\operatorname{Var}\left(D_{\text {norm }}\left(\varepsilon_{\tau}\right)\right)=\frac{K_{1}}{S N R}\left(1+\frac{K_{2}}{S N R}\right)
$$

$$
\begin{aligned}
K_{1} & =\frac{9 \operatorname{sinc}\left(\pi \beta \frac{\Delta}{2}\right)^{2}(1-\operatorname{sinc}(\pi \beta \Delta))}{4 . N_{P} \cdot K_{\text {norm }}{ }^{2}} \text { and, } \\
K_{2} & =\frac{9(1+\operatorname{sinc}(\pi \beta \Delta))}{32 N_{P} \operatorname{sinc}\left(\pi \beta \frac{\Delta}{2}\right)^{2}} .
\end{aligned}
$$

